\title{
Simulation of Agent Behavior in a Goal Finding Application
}

\author{
Sharad Sharma, Member, IEEE and Supriya Lohgaonkar \\ Department of Computer Science \\ Bowie State University \\ Bowie, MD 20715, USA \\ Email: \{ssharma, lohgaonkars0604\}@bowiestate.edu
}

\begin{abstract}
There has been increasing interest in simulation of agent behavior in the context of agent based modeling. This paper concentrates on the use of fuzzy logic in simulating agent based behavior. Our approach is decomposed into two levels. The higher level addresses the agent's goal finding behavior and lower level addresses collision detection and avoidance behavior. Our approach focuses on modeling individual behavior as well as group behavior. Individuals constantly adjust their behavior according to the dynamic factors in the environment. We hypothesize that people with similar characteristics such as race, age, and gender are more likely to collaborate with each other in order to reach a goal. This paper describes an agent based system implementation for crowd behavior. The simulation evaluates different evacuation and damage control decision making strategies beforehand, which allows the execution of the most effective evacuation scheme during real-time emergency scenario.
\end{abstract}

\section{INTRODUCTION}

Simulation of crowd behavior and human behavior modeling in response to manmade and natural disasters has become a key research field after September $11^{\text {th }}$ attack. Simulation of crowd behavior involves modeling the environment and the groups of people. An accurate simulation model of the emergency environment allows the responsible agencies to evaluate different evacuation strategies and damage control policies. Also, the simulation model could be used for education and training response for the people responsible for safety and evacuation strategies [1]. Construction of crowd behavior simulation is a complex task as it involves creation of both individual behavior as well as group behavior. To enhance the level of details, we propose to build a simulation model mimicking humans in the crowd based on intelligent agent paradigm. An intelligent agent is autonomous and has its own characteristics such as social ability, reactivity, cooperativeness, learning ability, and adaptability [2]. Our work focuses on modeling individual behavior and group behavior. Agents constantly adjust their behavior and goal according to the dynamic factors in the environment. We present an approach for goal finding behavior by using fuzzy logic in collision avoidance by using distance methodology.
The goal of our work is to produce a simulation of crowd behavior scenario where the goal is likely to change during the simulation. This study aims to investigate the effectiveness of the simultaneous and staged goal finding strategies under different path network structures using agent-based simulation. A more readily attainable goal would be to determine a threat environment easily using these parameters. Our work focuses on adaptive behavior and collective behavior where the agents are likely to cooperate with each other in order to reach a goal. Our hypothesis is that people with similar characteristics will collaborate with each other in order to reach a goal. We propose to develop a mechanism where agents have characteristics such as autonomy, social ability, cooperativeness, learning ability, and level of panic, which define their final behavior to reach a goal.

The rest of the paper is structured as follows. Section II, describes the previous work briefly. Section III, illustrates the system architecture of the proposed system. Section IV describes the fuzzy behavior, collision detection behavior, and obstacle avoidance behavior. Section V, shows the simulation of the proposed application. Section VI, lists conclusions and future work.

\section{RELATED WORK}

Agent-based modeling and simulation (ABMS) is an approach to modeling systems that contain autonomous agents. Agent based applications have grown in number lately due to advance in technology. ABMS originated from complex adaptive systems (CAS) and the underlying notion that "systems are built from the ground-up" [3]. CAS argues how complex behaviors originate in nature from myopic and autonomous agents. The field of CAS is motivated by investigations into adaptation and emergence of biological systems. John Holland, identified properties and mechanism common to all CAS systems [4].

- Aggregation: allows groups to form.

- Nonlinearity: invalidates simple extrapolation

- Flows: allow the transfer and transformation of resources and information 
- Diversity: allows agents to behave differently

These CAS properties provide a useful reference for the definition of agent-based models. Reynolds [5] described boids (birds ) simulation based on simple behavioral rules that lead to emergent and seemingly organized behavior. In his boids model, each agent has three rules that govern the movement of the agent [5]:

- Cohesion: each agent steers toward the average position of its nearby flockmates.

- Separation: each agent steers to avoid crowding local flockmates.

- Alignment: each agent steers towards the average heading of local flockmates.

The simple rules of boid behavior and agent interaction lead to the emergence of collective intelligence or swarm behavior. The natural systems adapt to the environment and effectively optimize their behavior over time. Swarm intelligence has lead to many optimization solutions, such as ant colony optimization that have been used to solve practical scheduling and routing problems [6]. We attempt to create an agent that has both individual characteristics as well as group characteristics. Most of the crowd simulations involve modeling crowd as a group of people with common characteristics and objectives. However, we provide a simulation framework where people with similar characteristics try to come together in order to reach a goal in emergency. This behavior is quite similar to boids behavior where the birds follow the leader and stay in the group. We have built the simulator in $\mathrm{C} \#$ for crowd movement where agents are shown in different colors. When the simulation starts the agents have an initial goal. The goal can be changed during the simulation to a different goal. Some agents have secondary goal that leads them to group together as they move towards the goal. Crowd simulation involves modeling environment and human behavior. A realistic representation of human behavior in a crowd simulation requires a model that is able to represent partly the complex human behavior. Human behavior is unpredictable when it comes to making decisions in emergency situations [7].

Fuzzy logic plays an important role in modeling human behavior when there is uncertainty involved. Model such as PETEEI (a PET with Evolving Emotional Intelligence) has been developed for simulating emotions in agents using fuzzy logic [8]. Another model, FLAME (Fuzzy Logic Adaptive Model of Emotions) has been developed for mimicking emotional behavior in intelligent agents through a computational model [9]. AvatarSim [10-14] has also been developed where fuzzy logic is used to refine human behavioral characteristics. AvatarSim incorporates two types of behavior attributes that are fuzzy behavior attributes and non-fuzzy behavior attributes. It uses fuzzy rules to simulate a mixture of panic and stress emotional behaviors. It is able to handle real-life scenarios, in which people behave differently, depending on their level of panic or stress attributes. Its applications vary from building evacuation [12], aircraft evacuations [14], and battlefield simulations [15].

\section{SYSTEM ARCHITECTURE}

System Architecture of the proposed application shows layered architecture of agent based system. Graphical User Interface (GUI) layer of the application includes both the simulation area and control buttons. The user interacts with the system through the user interface screen that has control buttons to start, stop and view step by step simulation. Application layer is responsible for decision making and planning. It is followed by the navigational layer that creates multiple agents and sets their destination. Algebraic layer is responsible for the declarations of variables and mathematical calculations. The proposed agent based system contains different behaviors like goal following behavior, obstacle detection, obstacle avoidance, group behavior, and fuzzy behavior.
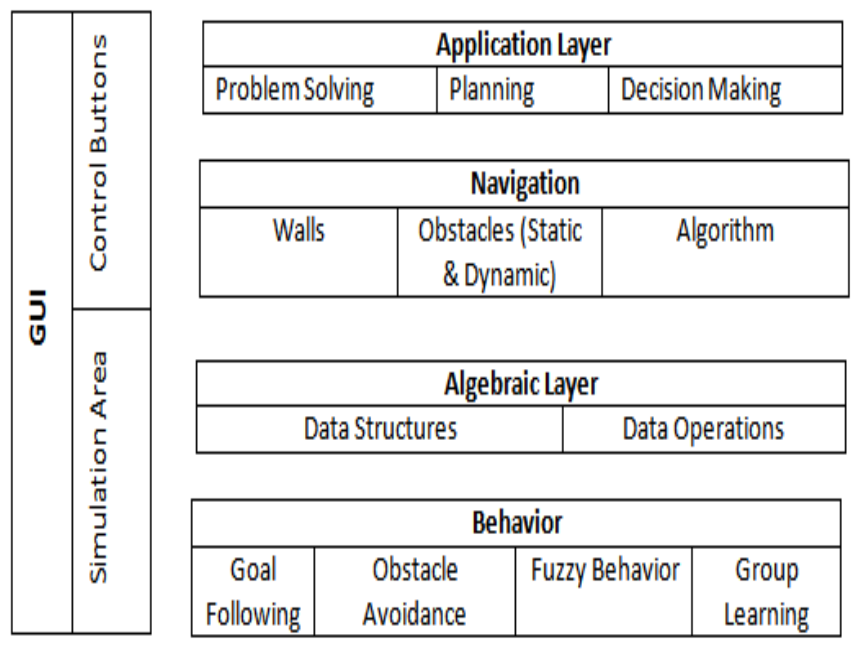

\begin{tabular}{|c|c|}
\hline \multicolumn{2}{|c|}{ Algebraic Layer } \\
\hline Data Structures & Data Operations \\
\hline
\end{tabular}

\begin{tabular}{|c|c|c|c|}
\hline \multicolumn{4}{|c|}{ Behavior } \\
\hline $\begin{array}{c}\text { Goal } \\
\text { Following }\end{array}$ & $\begin{array}{c}\text { Obstacle } \\
\text { Avoidance }\end{array}$ & Fuzzy Behavior & $\begin{array}{c}\text { Group } \\
\text { Learning }\end{array}$ \\
\hline
\end{tabular}

Figure 1. System Architecture

Figure 1 shows the system architecture of the proposed application that is developed in C\#. Fuzzy logic is used to find the direction of the agent to move toward the goal. Initially, the agents are assigned random positions in the environment. The aim of the agents is to reach the goal by avoiding obstacles in its path. Also, a secondary goal is assigned to some of the agents that leads them to stay in a group in order to reach the goal. Motion planning is done to avoid static obstacles or dynamic obstacles. The static obstacles are objects or walls in the environment, whereas the dynamic obstacles are other moving agents in the environment.

\section{Simulation OF AGENT BEHAVIOR}

Social behavior is a complex phenomena and it emerges from interactions among group of autonomous agents. The proposed application demonstrates social emergent phenomena including competitive, queuing, and cooperative behaviors. Figure 2 shows the flow chart for an agent to navigate around an obstacle to reach its goal. The agent calculates the path to move towards a goal by calculating its direction and angle by avoiding obstacles in between. The following behaviors are observed in the proposed application. 


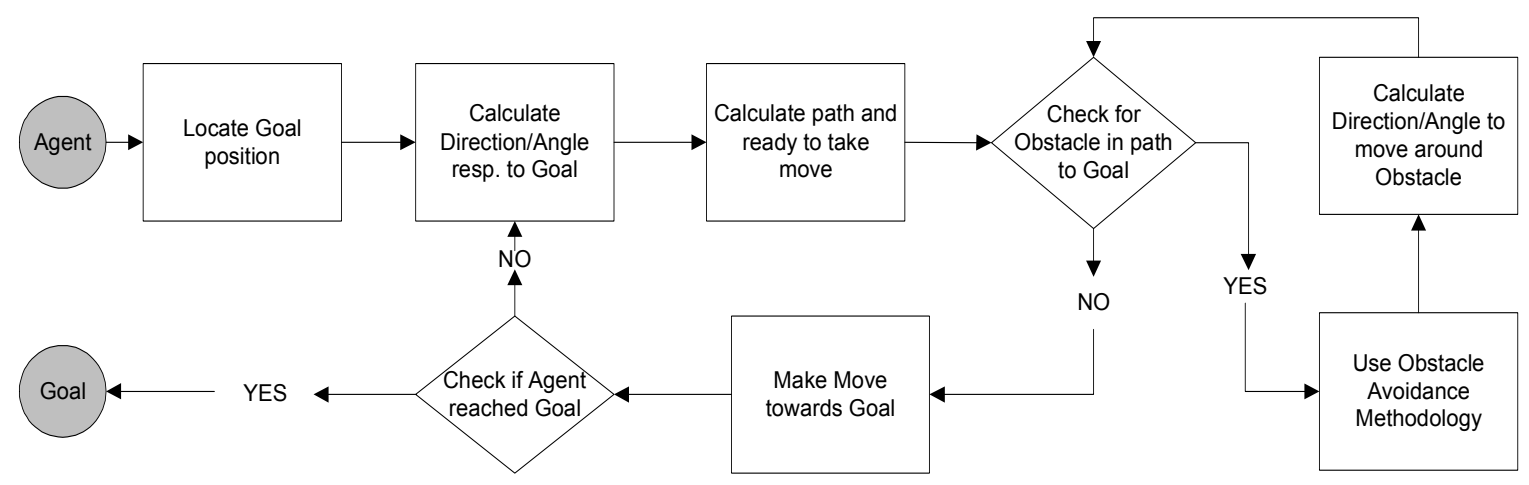

Figure 2. An agent moving towards a goal

\section{A. Fuzzy Behavior}

The fuzzy behavior is implemented to aid the agents in moving towards their goal. The goal finding behavior is implemented with the help of two linguistic variables i.e. angle and distance. The angle refers to the direction in which the agents will move. Distance is another linguistic variable which defines if the goal is at left or right or centre of the agent. With the help of linguistic variables fuzzy rules are defined as shown in the Table I below

TABLE I. FuZZY RULES

\begin{tabular}{|c|c|c|c|}
\hline $\begin{array}{c}\text { Distance } \\
\text { /Direction }\end{array}$ & Left & Centre & Right \\
\hline East & East-Left & East-Center & East- Right \\
\hline West & West-Left & West-Centre & West- Right \\
\hline North & North-Left & North- Centre & North- Right \\
\hline South & South- Left & South- Centre & South- Right \\
\hline
\end{tabular}

The membership function takes crisp value as parameter and output number between 0 and 1 . Each linguistic value has a associated member function. The membership functions define crisp value for specific linguistic value. e.g. "left", new TriangleMembershipFunction(-4000, -10, 0). Fuzzy sets are defined by using linguistic variable and crisp value.

FuzzySet(Variable variable, double crispValue)

Fuzzy Rules are basically if $<$ conditions $>$ then $<$ condition $>$

If direction $=$ north and direction $=$ west then turn $=-15$.

The crisp value is assigned from given conditions.

\section{B. Collision Detection Behavior}

The proposed system uses distance-based collision detection method. This involves using distance between two objects to determine wheather the agents have collided. This method favors perfect cicles but can be improved for other shapes too. Figure 3 shows a pair of circles as agents. The distance between agent and obstacle is 50 , which is the sum of their radius.

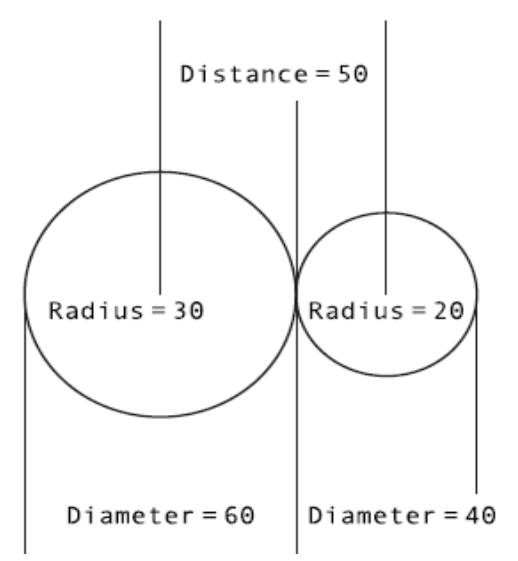

Figure 3. The distance between two agents

$(\mathrm{x} 1, \mathrm{y} 1)$ are the coordinates of the first agent and $(\mathrm{x} 2, \mathrm{y} 2)$ are the coordinates of the obstacle.

$$
\begin{aligned}
& d x=x 1-x 2 \\
& d y=y 1-y 2
\end{aligned}
$$

Collision (static and dynamic) is checked for an agent moving toward a goal. When a safe distance value between obstacle and goal goes below the threshold value, the obstacle avoidance method is called to avoid the obstacle.

\section{Obstacle Avoidance Behavior}

As shown in Figure 4, the big circle denotes the obstacle. The system considers a virtual circle outside obstacle circle and divides the perimeter of the virtual circle into 12 equal parts. These 12 points are used to rotate the agent on its perimeter without collision with an actual obstacle. Distance of nearest point from an agent is calculated. The agent then moves to the nearest point and then the next nearest point and thus avoids collision with the obstacle. When an agent completely avoids an obstacle, it recalculates its angle and direction with respect to the goal. It checks for possible collision with obstacles or with other agents before it makes a move and continues to do so until it reaches the goal. 


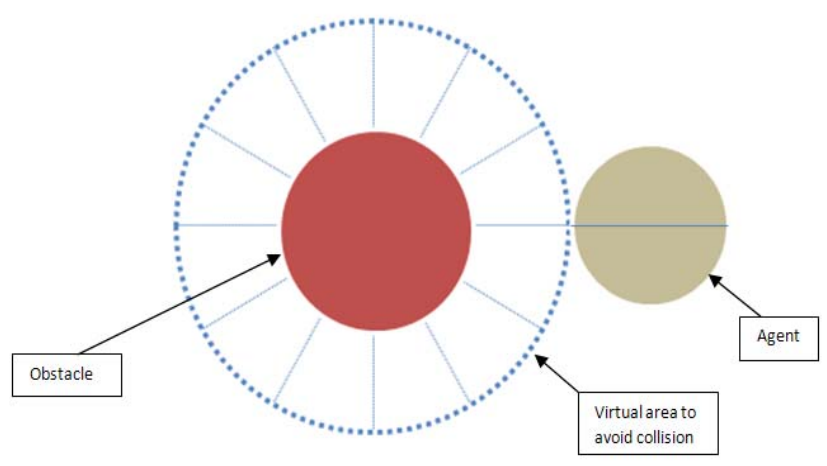

Figure 4. area around an obstacle to avoid collision

\section{SimUlation}

Each agent calculates its direction and angle with respective to its goal. When the simulation starts the agents start moving toward the goal. The green colored circles are the static obstacles. If the obstacle comes in path of an agent, it tries to avoid the obstacle by calculating alternate route. Thus, agent goes away from obstacle by avoiding collision. Once the agent reaches its goal it becomes invisible. Thus, simulation run until all the agents reaches to the goal.

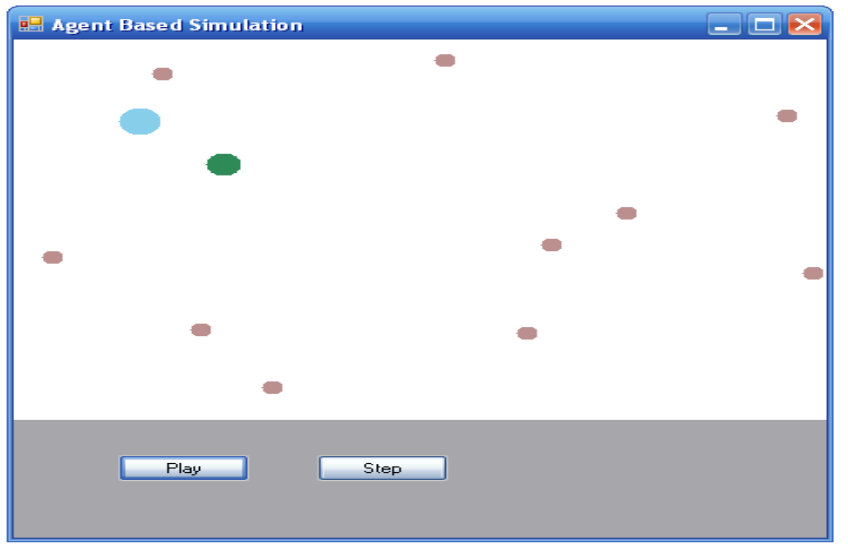

Figure 5. The proposed application devloped in C\#

Figure 5 shows position of the agents and the obstacles in the simulation. The blue colored circle denotes the goal and its position could be changed during the simulation. Green colored circles are static obstacles and their position remains the same for the life of simulation. The brown colored circles are the agents. In the bottom part, buttons displayed are "Play", "Step" and "Stop". Play button is for starting a simulation, stop button is to stop the simulation and step button is used to see step by step movement of all the agents. Fuzzy rules are defined to find out the angle and direction of each agent that hasn't reached its goal. The agents are assigned to random position in the environment when they are rendered for the first time. During each simulation step the agents locate position of goal, calculate angle/distance, and move towards the goal. Thus, agents show goal finding and obstacle avoiding behavior.

\section{CONCLUSIONS}

The simulation aids in establishing a direct relationship between population of the crowd and causalities. It also helps in providing an estimate of number of people reaching a goal in case of fire when the goal is likely to change dynamically during the evacuation process. The application developed by the authors is able to simulate the cooperative behavior and navigational behavior through the use of fuzzy logic. This application could be used as training and education as it allows evaluating different evacuation and damage control decision making strategies beforehand. The future work will involve the implementation of altruistic behavior and selfish behavior. Moreover, the fuzzy logic behavior will be extended to include more behaviors such stress, anger, etc.

\section{REFERENCES}

[1] S. Jain and C. R. McLean, "An Integrated Framework for Modeling and Simulation for Incident Management", Journal of Homeland Security and Emergency Management, Volume 3, Issue 1, 2006.

[2] R. Laughery, "Computer simulations as a tool for studying humancentered systems", Proceedings of the 2001 winter simulation conference, pp. 61-65, 1998.

[3] N. R. Jennings, "On agent-based software engineering", Artificial Intelligence, 117:277-296, 2000.

[4] J. H. Holland, "Hidden order: how adaptation builds complexity", Reading, MA: Addison-Wesley, 1995.

[5] Craig Reynolds, 2006. Boids. <http://www.red3d.com/cwr/boidss/>.

[6] E. Bonabeau, M. Dorigo and G. Theraulaz., "Swarm intelligence: from natural to artificial systems", Oxford: Oxford University Press, 1999.

[7] S. Sharma, "Modeling and simulation of multi-agent systems for emergency scenarios," PhD thesis, Department of Electrical Engineering and Computer Engineering, Wayne State University, Detroit, December 2006

[8] Magy Seif El-Nasr Thomas R. loerger John Yen, PETEEI: A PET with Evolving Emotional Intelligence, Autonornous Agents '99, ACM 1999.

[9] El-Nasr, Yen, and Ioerger, FLAME - Fuzzy Logic Adaptive Model of Emotions, International Journal of Autonomous Agents and MultiAgent Systems, in press, 1999.

[10] S. Sharma, "Avatarsim: A Multi-Agent System for Emergency Evacuation Simulation", 17th International Conference on Software Engineering and Data Engineering, Los Angeles, California USA, June 30 - July 2, 2008.

[11] S. Sharma, "Simulation and modeling of group behavior during evacuation", IEEE Symposium Series on Computational Intelligence, Intelligent Agents, March 30-April 2, Nashville, TN, USA, 2009.

[12] S. Sharma, and H. Singh, "Multi-agent system for simulating human behavior in egress simulations," Proceedings of NAACSOS, Annual Conference of the North American Association for computational social and organizational sciences, Notre Dame, Indiana, June, 2006.

[13] S. Sharma, A. Awasthi, "Fuzzy approach for forecasting navigation in simulating agent based behavior", Proceedings of Parallel and Distributed Computing Conference, ISCA-PDCS, Sept 2005.

[14] S. Sharma, H. Singh, A. Prakash, "Multi-agent Modeling and Simulation of Human Behavior in Aircraft Evacuations", IEEE Transactions on Aerospace and Electronic Systems, Vol. 44, number 4, Oct 2008.

[15] S. Sharma, S., H. Singh, G.R. Gerhart, "Simulation of convoy of unmanned vehicles using agent based modeling, Procedings of SPIE Conference on Security and Defense, Florence, Italy, Vol. 6736 pp. 673605-1-673605-8, 17 - 20 September 2007. 